\title{
Nuevas aportaciones al estudio del recinto de la judería de Valencia delimitado en 1244
}

Ma Concepción López González*

Universidad Politécnica de Valencia

\begin{abstract}
A raíz de la conquista de la ciudad, el rey Jaime I delimita en 1244 en Valencia un espacio para los judíos. Con esta finalidad, hace referencia a cinco puntos de los que, hasta el momento, solo se conocía la ubicación exacta de la puerta de la Xerea. El análisis de las propiedades limítrofes con estos hitos en los listados de los registros I y III del Llibre del Repartiment ha permitido determinar la ubicación de los otros cuatro puntos, pudiendo comprobar que los baños de Amnalmelig se encontraban al sur del barrio judío contrariamente a lo que, hasta ahora, se había pensado. Los resultados de la investigación se han plasmado sobre planos actuales, reconstruyendo el urbanismo de la época basándose en la cartografía más antigua de la ciudad (Mancelli [1608] y Tosca [1704]), en informes arqueológicos y en los vestigios de calles actualmente desaparecidas que han pasado a formar parte de propiedades privadas.
\end{abstract}

Palabras Clave: Juderías; Repartiment; Juheria Vella; planimetría; toponimia; baños de Amnalmelig; cultura material.

New Contributions to the Study of the Jewish Quarter of Valencia Delimited in 1244. - In 1244 King James I of Aragon determined the area of the Jewish Quarter of Valencia making reference to five points of the city of which, until now, only the exact location of the Xerea Gate was known. The analysis of the bordering properties with milestones in the lists of the Records I and III of the Llibre del Repartiment has allowed us to determine the location of the other four points, making it possible to verify that Amnalmelig's baths were to the south of the Jewish Quarter, contrary to current opinion. The results of the research have been captured on plans that reconstruct the urban planning of the epoch, based on the most ancient cartography of the city (the plans of Mancelli [1608] and Tosca [1704]), on archaeological reports and on the remains of streets that were abolished to form part of private properties.

KeYwords: Jewish Quarter; Repartiment ('Repartition Book'); Juheria Vella; Maps; Jewry Gazetteer; Amnalmelig Baths; Material Culture.

"mlopezg@ega.upv.es 
Ya nada queda en el urbanismo de la Valencia del siglo XXI que recuerde la antigua trama urbana de la judería medieval. Las intervenciones en el área ocupada por el antiguo barrio judío, agresivas en muchos casos, se han sucedido a lo largo de los años. Las primeras alteraciones urbanísticas importantes se llevan a cabo en el siglo XIV como consecuencia del violento ataque perpetrado a la judería en 1391 por cristianos enaltecidos. El barrio quedó totalmente arruinado, y en ese momento, los jurados aprovechan para incorporar reformas urbanas destinadas a facilitar la comunicación entre el centro de la ciudad y el camino hacia el puerto marítimo: se materializa la apertura de la calle del Mar $^{1}$ atravesando lo que había sido el espacio de los judíos.

Durante el siglo XVI se levanta el Estudio General (Universidad) y comienza la construcción (1586) del Real Colegio del Corpus Christi, edificado sobre el solar de cuarenta y nueve casas y algunas calles compradas por el Patriarca San Juan de Ribera ${ }^{2}$. Ambos edificios, de considerables dimensiones, afectarán a extensas áreas urbanas incluidas en el recinto judaico con la consiguiente desaparición de ciertas calles, datadas en escrituras de compra-venta y de las que no es posible determinar con exactitud su localización. En el siglo XVII se cierran algunas vías como la de Christofol Soler y se abren otras como la de San Carlos, actualmente denominada de Muñoz Degrain. En el siglo XIX se lleva a cabo la apertura de la calle de la Paz y se derriban los conventos de San Cristóbal y Santa Tecla, lo que supuso una importante intervención en el antiguo entramado urbano. Por último, durante el siglo XX, se materializa la calle del Poeta Querol, derribando para ello muchos de los antiguos palacios y caserones que aun se conservaban y dando lugar a la desaparición de pequeñas plazuelas y estrechas calles que en su día formaron parte de la judería.

Este breve recorrido por los avatares urbanísticos que sufre el barrio donde se ubicaba la judería pone de manifiesto la escasez de vestigios que den luz sobre la antigua trama urbana que conformaba el barrio judaico.

\footnotetext{
${ }^{1}$ Esta calle se abrió en 1412 para favorecer la comunicación entre el centro de la ciudad y la salida hacia el puerto marítimo: «cap jusá a la plaça de la Figuera tro al portal de la Exerea», cf. José Hinojosa Montalvo, En el nombre de Yahveh. La judería de Valencia en la Edad Media (Valencia, 2007), 427.

${ }^{2}$ Francisco Danvila Collado «Clausura y delimitación de la judería de Valencia de 1390», Boletín de la Real Academia de Historia 18 (1891), 142-158: 147.
} 
El objetivo inicial de esta investigación era establecer una planimetría real de la judería valenciana, utilizando para ello los planos más antiguos de la ciudad, Mancelli (1608) y Tosca (1704), adaptados al catastral actual y representando sobre los mismos los datos documentales extraídos del Llibre del Repartiment, protocolos notariales e informes arqueológicos. En el transcurso de la misma se ha llevado a cabo un profundo análisis del Llibre del Repartiment como fuente fundamental para el conocimiento del urbanismo de la época. A la luz de este documento se han descubierto datos novedosos referentes a la delimitación del primer recinto judío delimitado en 1244 y de los barrios circundantes. Asimismo, las excavaciones arqueológicas y la demolición de ciertas construcciones en el palacio de Valeriola (donde se encontraba la carnicería judía y pasaba el muro de cierre) y en el cementerio medieval de San Juan del Hospital, junto a la investigación llevada a cabo para la puesta en valor del espacio cementerial de los Sanjuanistas ${ }^{3}$, han conducido al descubrimiento de ciertas vías urbanas que han desaparecido como tales, pero que han perdurado formando parte de propiedades privadas.

En el Llibre del Repartiment ${ }^{4}$ se recogen datos relativos a las donaciones realizadas por el rey Jaime I a todos aquellos que, de un modo $\mathrm{u}$ otro, colaboraron en la conquista o vinieron como pobladores. Este documento está dividido en tres registros: En el registro I se anotan las donaciones de casas en la ciudad de Valencia agrupándolas por los años en que estas fueron realizadas. Este documento tiene un gran valor porque en él quedan reflejados en muchos casos el atzucac o "callejón' ${ }^{5}$ musulmán en que se ubicaba la casa, así como el nombre del nuevo y antiguo propietario y el nombre de los propietarios que lindan con la misma. Desgraciadamente, dependiendo de la rigurosidad del escribano, existen casos en que solo se hace referencia a la donación de «unas casas en la ciudad» consignando únicamente el nombre de la

\footnotetext{
${ }^{3}$ Este proyecto fue realizado en el año 2005 por los arquitectos Jorge García Valldecabres y Concepción López González.

${ }^{4}$ Para esta investigación se ha utilizado la edición dirigida por Antoni FerRANDO I FRAnCÉs, Llibre del Repartiment de València (València, 1979).

${ }^{5}$ Federico Corriente CóRdoba, Diccionario de arabismos y voces afines en iberorromance (Madrid, 2003), 243.
} 
persona a quien se hace la donación sin anotar más información sobre su enclave. En el registro II se anotan las donaciones ubicadas fuera de la ciudad y añade un listado de los hornos, molinos y obradores que el rey ha donado o ha cedido en alquiler a los nuevos habitantes de Valencia. En el registro III se hace un recuento de las casas de la ciudad de Valencia agrupándolas por partidas según el origen de los nuevos pobladores. La utilización de estos tres documentos relacionando entre sí los datos que contiene cada uno de ellos permite comprobar el atzucac ('callejón'), barrio o partida donde se ubicaba una casa y el lugar que el mismo ocupaba dentro de la ciudad. Esta ha sido la clave para determinar la situación de los baños de Amnalmelig y el horno de Abinulliz como se verá más adelante.

Francisco Danvila Collado ${ }^{6}$ (1891) y José Rodrigo Pertegás (1913), cuyos estudios quedan resumidos en el opúsculo que este último escribe para José Sanchis Sivera ${ }^{7}$, son los primeros autores que, basándose en datos extraídos de archivos y protocolos notariales, plantean un esquemático plano del cierre y delimitación de la judería de Valencia. Han sido un referente para todos aquellos investigadores que se han aventurado en el estudio de este desaparecido barrio judío. Anteriormente Orellana ${ }^{8}$ (1791) planteó una delimitación muy indefinida del barrio y posteriormente Carboneres ${ }^{9}$ (1873) propuso otra

\footnotetext{
${ }^{6}$ Danvila Collado «Clausura y delimitación de la judería de Valencia en 1390», 142-158.

${ }^{7}$ Este opúsculo fue escrito a instancias del historiador Sanchis Sivera como apéndice del libro La iglesia Parroquial de Santo Tomás. En 1913 fue editado de forma independiente con el título La judería de Valencia con una tirada de cincuenta ejemplares.

${ }^{8}$ Marcos Antonio Orellana, Valencia antigua y moderna (Valencia, 1923; reimpr. Valencia, 1985), tomo I, 281: «[...] lo era el ámbito que hay desde inmediato a Santa Tecla, yendo por la calle del mar, toda la acera a mano derecha hasta llegar a la actual Plaza de la Congregación internándose dicha línea hacia el Colegio del Patriarca, en cuya demarcación se comprende el actual convento de San Christoval, Plaza de la Cruz Nueva \&c.».

${ }^{9}$ Manuel Carboneres y Quiles, Nomenclátor de las puertas, calles y plazas de Valencia (Valencia, 1873; reimpr. Valencia, 1980), 14: «Desde la puerta situada en la plaza de la Figuera corría el muro por la calle de las Avellanas a la derecha de dicha calle y por la del Milagro seguía hasta San Juan del Hospital, dejando aislada esta iglesia; salía a la calle Trinquete de Caballeros, y por dentro de la Congregación iba a unirse a la puerta de la Exerea; desde esta puerta seguía por la calle de la
} 
en la que extendía el espacio judío, referido a la ampliación de 1390, hasta la calle del Milagro ${ }^{10}$. Otros autores como Lacave ${ }^{11}$, Sanchis Guarner $^{12}$, Lerma ${ }^{13}$, Teixidor y Boira ${ }^{14}$, Hinojosa Montalvo ${ }^{15}$, Magdalena Nom de Déu ${ }^{16}$, Aldana Fernández ${ }^{17}$, Roca Traver ${ }^{18}$, entre otros, han descrito delimitaciones de la judería tomando todos ellos como referente las aportaciones de Rodrigo Pertegás ${ }^{19}$.

Cullereta, plaza de las Comedias, atravesando la calle de la Nave a la Universidad, a cuyo extremo estaba la puerta de En Esplugues: seguía luego por la calle del Hospital de Pobres Estudiantes, Horno de les Rates, plaza del Patriarca, calle de Libreros, plaza Villarrasa, calle de Cardona y por la plaza y calle de Luis Vives a unirse a la puerta de la Figuera, punto de partida».

${ }^{10}$ No es posible que el barrio judío llegara hasta la calle del Milagro ya que esta calle no fue abierta hasta 1393: «[...] del cap d'aquel atzucac tro al dit carrer iusa no hagues pus espay sino alcuns patis vells e podrits e de grau legea, sens tot profit de Sant Joan del Espital, qui no fallen fruti sino a rates e aranyes» (18-VII-1393, Archivo Municipal de Valencia [= AMV], llm g3-5, fol. 206r).

${ }^{11}$ José Luis LaCAVE, Juderías y sinagogas españolas (Madrid, 1992), 63-64.

${ }^{12}$ Manuel SAnchis GuARNER, La ciutat de València (València, 1976), 102 y 152.

${ }^{13}$ Vicent LERMA, «La segregación espacial de las minorías: la judería y la morería», en Historia de Valencia, fasc. 12: La expansión de la ciudad medieval (Valencia, 1999), 144.

${ }^{14} \mathrm{M}^{\mathrm{a}}$ Jesús Teixidor de OtTo y Josep Vicent Boira i MARQuÉs, El entorno urbano de la Universidad. Sapientia Aedificavit: Una biografia de l'Estudi General de la Universitat de València (Valencia, 1999), 164-165.

${ }^{15}$ Hinojosa Montalvo, La judería de Valencia en la Edad Media, 415-444.

${ }^{16}$ Jose Ramón Magdalena Nom DE Déu, «Un zoco judaico en la Valencia medieval (1351-1389)», Sefarad 39 (1979), 309-331.

${ }^{17}$ Salvador Aldana FernándeZ, Los judíos de Valencia: un mundo desvanecido (Valencia, 2007), 80-216.

${ }^{18}$ Francisco Roca Traver, Los judios valencianos en la Edad Media (Valencia, 1998), 106.

${ }^{19}$ José Rodrigo Pertegás, La judería de Valencia (Valencia, 1913; reimpr. Valencia, 1992), 8: «Partiendo de la puerta de la Xerea, pasaría junto a San Juan del Hospital, por las casas que actualmente son de la plaza de la Congregación y, por junto al cementerio de este hospital, llegaría al baño de Nicholau Tamarit, que estaba tal vez en la actual calle del Torno de San Cristóbal; atravesaría la manzana de esta calle, en dirección paralela y, por sitio intermedio a las del Milagro y del Mar, hasta la de las Avellanas; por esta seguiría a la plaza de la Figuera, próxima a la actual de la Reina, donde se encontraba la puerta principal, y desde aquí buscando la calle del Ave María, torcería por la que últimamente se llamó del Gallo, hasta la plaza dels Cabrerots, donde había otra puerta, y de aquí, por la actual calle del Pollo o sus cercanías, a encontrar la muralla que seguía hasta la puerta de la Xerea». 


\section{1.- El PRIMER RECINTO DELIMITADO POR JAIME I EN 1244 Y SU POSTERIOR AMPLIACIÓN EN 1273}

Anteriormente a la conquista de la ciudad por Jaime I en 1238 ya existía una comunidad judía en Valencia agrupada en un barrio como demuestran las donaciones de casas que el rey Jaime I otorga el 5 de septiembre de 1238 a Artal de Foces ${ }^{20}$ in vico judeorum o las donadas el 15 de diciembre de ese mismo año a dos judíos gerundenses (Cresques y Salomón), llegados de Bellcaire y de Girona respectivamente, in calle judarie ${ }^{21}$. El Llibre del Repartiment también usa otros términos para referirse al área habitada por los judíos como Coilo ${ }^{22}$, Calle $^{23}$, o Toll $^{24}$, es decir, el Call.

Como barrio lo identifica Jaime I cuando ordena el 20 de octubre de 1244 su delimitación ${ }^{25}$. En la misma señala cinco hitos de la época que sirven de puntos de referencia para establecer el recorrido que debe tener el muro de cierre del barrio judaico: el adarve Abingeme, el baño de Amnalmelig, la puerta de la Exarea, el horno de Abinulliz y el adarve de Abrahim Alvalençí.

De todos ellos solo está documentada la ubicación de la puerta de la Xerea que Ortí y Mayor ${ }^{26}$ sitúa perfectamente en el acceso principal a la iglesia de

${ }^{20}$ Llibre del Repartiment, reg. I, fol. 45v.

${ }^{21}$ Llibre del Repartiment, reg. I, fol. 51v.

${ }^{22}$ «Gil, domos de (Almantel) de Amodarif in Coilo» (donación del 13 de junio de 1238), Llibre del Repartiment, reg. I, fol. 25.

${ }^{23}$ «Rodericus Didaci, notarius regis, domos de Aly Ambalagay quas habet in calle [...]» (donación del 25 de junio de 1238), Llibre del Repartiment, reg. I, fol. 26v.

${ }^{24}$ «Lupus Eximini Daçcots, miles, domos de Jucef Alharar in tollo [...]» (donación del 9 de enero de 1239), Llibre del Repartiment, reg. I, fol. 53v.

${ }^{25}$ «Judei in Valentia habitantes et habitaturi [totum] illum barrium sicut incip[it] del Adarp Abingeme usque ad balneum de Amnalmelig et ab isto loco usque ad portam de Exarea et ab hac porta usque ad furnum de Abinulliz et usque ad Adarp Abrahim Alvalençi et volumus quod habeant et populentur secundum forum [et] consuetudinem algeme Barchinone. XIII kalendas novembris» (20 de octubre de 1244), Llibre del Repartiment, reg. I, fol. 80.

${ }^{26}$ «Esta puerta aún la habemos alcanzado y visto, pues se derribó lunes 16 de Diciembre, año de 1727, para la nueva fábrica del sumptuoso Templo de la Real Casa y Congregación del oratorio de San Felipe Neri [...]. Estaba este Arco (que era muy elevado) en el propio sitio en que ahora se ve la puerta principal de dicho Templo [...] de manera que el un pie, o estribo, de la Puerta de la Xerea se veía, como lo pudimos observar, dentro de lo que hoy es iglesia, y el otro pie estaba fuera, delante de las tres gradas que hay en la calle (ORTí Y MAYOR, «Fiestas 
Santo Tomás y San Felipe Neri aunque ni Tosca (1704) ni Mancelli (1608) la grafían en sus respectivos planos (actual plaza de San Vicente Ferrer).

Los baños de Amnalmelig fueron identificados inicialmente por Danvila con los denominados baños del Almirante ${ }^{27}$. Posteriormente, tras los estudios realizados para la recuperación de este emblemático edificio, se ha podido comprobar que se trata de unos baños de origen cristiano de factura posterior a la conquista de la ciudad. Rodrigo Pertegás los identificó con los baños de Nicolás Tamarit, situándolos «cerca de la calle que iba a la iglesia de San Cristóbal, que ya no existe» ${ }^{28}$ al norte de la judería, exponiendo en su opúsculo que se trata de una hipótesis y que «tendría gran importancia poder deslindar» ${ }^{29}$. Precisamente este es uno de los objetivos de esta investigación.

Para la localización de estos baños se han buscado las referencias a los mismos que se encuentran en el registro I del Llibre del Repartiment, a través de las cuales se ha tenido conocimiento de los nombres de los propietarios de las casas colindantes. Estos nombres se han localizado en los listados del registro III, donde las donaciones se agrupan por barrios para conocer aquel al que pertenecían y de este modo poder ubicar los baños en el entorno inmediato de la judería. Posteriormente se ha localizado este barrio sobre el plano de la ciudad a través de la información relativa a las lindes que ofrece el registro I.

El plano se ha delineado tomando como base el parcelario actual, sobre el que se han reconstruido los planos de Mancelli y de Tosca, por ser los más antiguos de la ciudad. La puerta de la Xerea y la muralla árabe se han grafiado de acuerdo a los planos realizados por Nicolau-Primitiu Gómez Serrano $^{30}$ de las excavaciones llevadas a cabo en la actual plaza de San Vicente Ferrer, por donde pasaba el valladar y la muralla y además se encontraba la puerta de la Xerea. Esta disponía de barbacana según

centenarias» [Valencia, 1740] cit. Teodoro Llorente, Valencia. Sus monumentos y artes. Su naturaleza e historia [Barcelona, 1887; reimpr. Valencia, 1980], tomo I, 487).

${ }^{27}$ Danvila Collado, «Clausura y delimitación de la judería de Valencia de 1390», 145.

${ }^{28}$ Rodrigo Pertegás, La judería de Valencia, 6.

${ }^{29}$ Rodrigo Pertegás, La judería de Valencia, 6.

${ }^{30}$ Nicolau-Primitiu Gómez Serrano, «D'arqueologia: excavacions de Valencia [1]», Anales del Centro de Cultura Valenciana 3 (1929), 75-96: 86. 
figura en el texto de la cesión a P. de Osca de un obrador «contiguo a la barbacana» de la puerta de la Xerea ${ }^{31}$.

Las torres ubicadas en el interior del palacio de los condes de Almansa en la calle Trinquete de Caballeros se han dibujo de acuerdo a los datos extraídos del informe arqueológico realizado en esta parcela.

La parcela de San Juan del Hospital de ha delineado con arreglo a las conclusiones extraídas de la investigación llevada a cabo para la puesta en valor del espacio cementerial sanjuanista expuestas en el proyecto de recuperación llevado a cabo por los arquitectos Jorge García Valldecabres y Concepción López González y en el libro El cementerio medieval de San Juan del Hospital ${ }^{32}$.

La calle Xepolella se ha grafiado con la inclinación que tuvo inicialmente y que viene marcada por los restos que aún se conservan del muro de cierre del recinto sanjuanista. Las desaparecidas calles de Cristòfol Soler, Çabateria y Micer Johan Davella han quedado atrofiadas en el parcelario formando parte de patios interiores por lo que su representación es la que marca el plano catastral actual, además de haber sido descubiertas formando parte del palacio de Valeriola y de las casas colindantes. También la calle Maçana es claramente reconocible en los planos de Mancelli y Tosca.

Así ha sido posible ubicar los baños de Amnalmelig que paso a detallar: Existen seis referencias a estos baños ${ }^{33}$ en el registro I situándolos limítrofes al Call judío que existía antes de la conquista como demuestra una donación de casas a dos judíos situadas en la judería frente a los baños ${ }^{34}$. Limitaban con las casas de Jucef, alfaquí ('médico') de Tortosa ${ }^{35}$,

${ }^{31}$ «P. de Osca, Operatorium contiguum barbachane porte Exeree» (27 de mayo de 1247), Llibre del Repartiment, reg. II, fol. 83v.

${ }^{32}$ Concepción López González y Jorge García VAlldeCabres, El cementerio medieval de San Juan del Hospital (Valencia, 2003).

${ }^{33}$ Llibre del Repartiment, reg. I, fols. 48, 50, 67, 73, 80 y 87v.

${ }^{34}$ «Salomon et Jofa, judei, domos in calle judeorum, quas tenent ante balneum de Almeli» (24 de julio de 1239), Llibre del Repartiment, reg. I, fol. 73.

${ }^{35}$ «Jucet, alfaquimus Dertusensis, domos de Azmet Albesqueri iuxta balneum de Avenmelich» (16 de septiembre de 1238), Llibre del Repartiment, reg. I, fol. 48. 


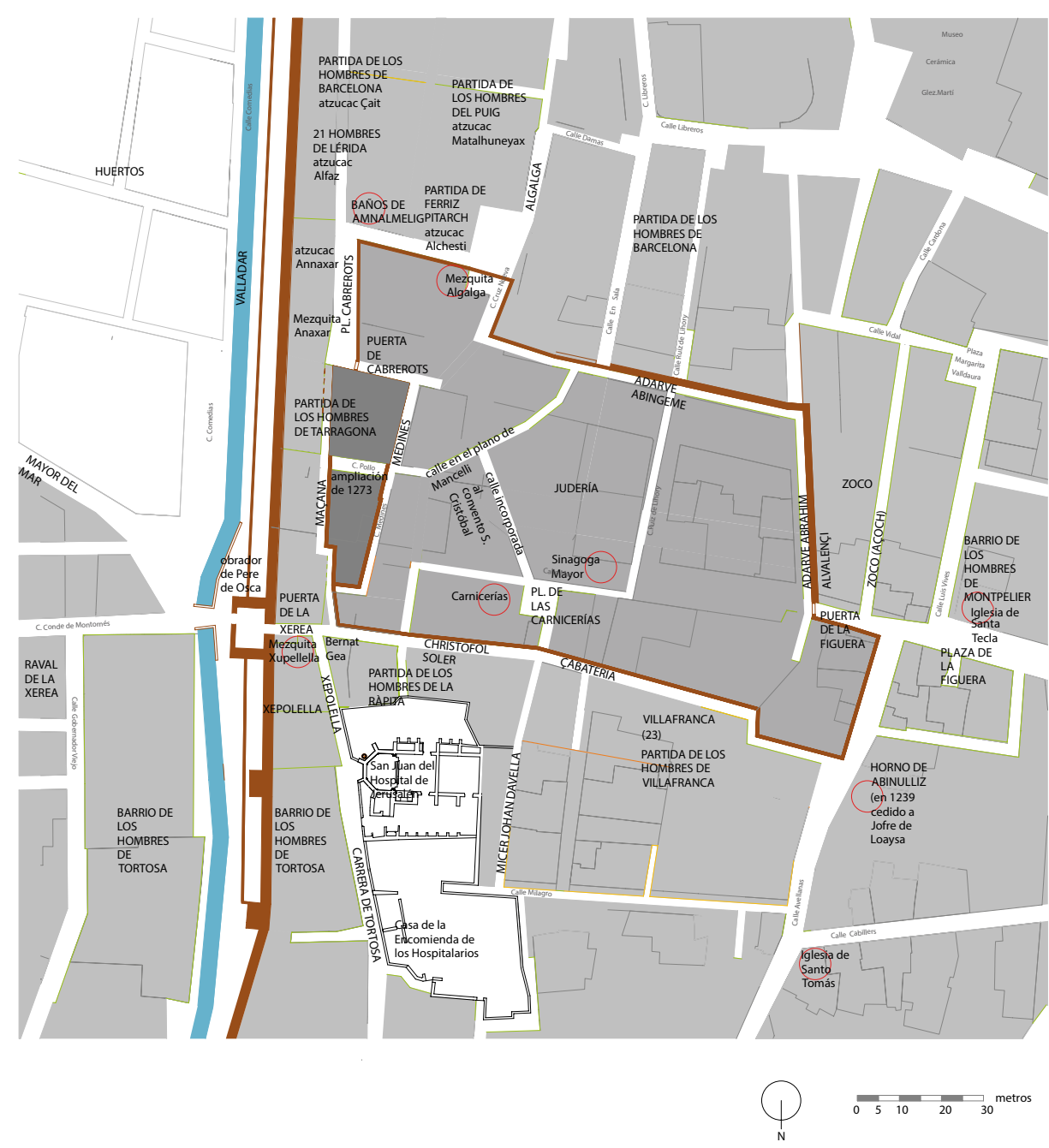

Fig. 1. Delimitación de la judería en 1244 (y ampliación de 1273) sobre el parcelario actual y los planos de Mancelli (1608) y Tosca (1704). Se indican los cinco hitos de la delimitación y barrios circundantes. Reproducción en color del plano en la edición electrónica de este artículo (http://sefarad.revistas.csic.es). 
Pere Vilaragut ${ }^{36}$, Bernat Macruç, Pere Olivella y Guillem de Aguiló ${ }^{37}$. Para conocer en qué barrio se encontraban estas propiedades y, consecuentemente los baños, se han buscado estos nombres en los listados de casas agrupados por partidas del registro III. Ninguno de ellos aparece en estos recuentos excepto el de Guillem de Aguiló que tiene unas buenas casas que pertenecían a Albogadin situadas en el atzucac Alchesti (Partida de Ferriç de Pitarch $)^{38}$, es decir, que los baños de Amnalmelig se encontraban al lado de este atzucac $^{39}$.

El siguiente paso es determinar la localización del atzucac Alchesti.

Consultando nuevamente el registro III se halla que el atzucac Alchesti lindaba con el atzucac Matalhuneyax donado a 20 hombres del Puig ${ }^{40}$.

Para conocer la ubicación de este último se han buscado en el registro I referencias sobre la donación a los hombres del Puig comprobando que este atzucac era colindante con el atzucac Çayt habitado por los hombres de Barcelona ${ }^{41}$. Es sabido que los hombres de Barcelona tenían su partida en el área de la ciudad limitada entre la puerta de Boatella y la de la Xe-

\footnotetext{
${ }^{36}$ «P. Vilaragut \miles/, domos de Aven Alavely iuxta balneum de Avinmelich» (27 de septiembre de 1238), Llibre del Repartiment, reg. I, fol. 50.

${ }^{37}$ «B. Macruç, domos de Fat Aberraya, que afrontant in balneis de Abenmalich et in domibus Petri de Olivela et in via et in domibus G. de Aquilone» (13 de mayo de 1239), Llibre del Repartiment, reg. I, fol. 67.

${ }^{38}$ «In partita Feriz de Pitarc nominata açuqaq alchesti [...] Ferriç de Pitarc, domos Lobreeccati. Guillem Sancti Petri, miles. G. de Agilo, domos bonas Abolgadin. Remanent II parvas», Llibre del Repartiment, reg. III, fol. 10v.

${ }^{39}$ En este caso, la palabra árabe az-zuqaq se refiere a un barrio y no a un callejón sin salida, acepción que le dieron posteriormente los cristianos. Podía disponer de puerta de acceso y vivir en él una o varias familias.

${ }^{40}$ «In partita Feriz de Pitarc nominata açuqaq Alchesti: VIII(I) domos et duas / P. Martinez Dago unas bonas domos Alchesti: II / Hominum de Podio: I çucac Matalhuneyax: XX domos sunt ibi [...]», Llibre del Repartiment, reg. III, fol. 10v.

${ }^{41}$ Tras enumerar los nombres de 32 hombres se anota la donación de «[...] XL domos in barrio in de Abencalbo et in barrio de Jayç Çenady, et afrontant in ca[rra]ria de Algalga et in carraria de Alhadrami (et afrontant) ex una parte, in barrio XXI hominum de Ylerda et, ex alia, in domibus de adalylis qui fuerunt ad Podio, et, de alia, in muro civitatis et, de aliis partibus, in partida hominum Barchinone. III nonas madii» (5 de mayo de 1239), Llibre del Repartiment, reg. I, fol. 66.
} 
rea $^{42}$, es decir, al Sur de la judería. Por lo tanto los baños de Amnalmelig no se encontraban al Norte del barrio judío como se ha supuesto hasta el momento, sino al Sur.

Conocido este dato, ha sido posible aproximar la ubicación de los baños a la manzana urbana donde se encontraban continuando con la información que proporciona el Llibre del Repartiment:

El atzucac Çayt, como se ha expuesto anteriormente, además de encontrarse junto a la partida de los hombres del Puig, también lindaba con la muralla y con el atzucac Alfaz donado a 21 hombres de Lérida. Según figura en el registro $\mathrm{I}^{43}$ este último lindaba al Sur con el anteriormente mencionado atzucac Çayt, con la muralla de la ciudad, con las casas de Azmeti y con el atzucac Annaxar perteneciente a la partida de los hombres de Tarragona que también lindaba con la muralla.

En el atzucac Annaxar se encontraba la mezquita del mismo nombre donada a Andreu de Odena $^{44}$ y por ella comienzan a contar las casas de la partida de Tarragona ${ }^{45}$ que figuran en el listado del registro III. Se consignan 91 casas para tarraconenses y, a renglón seguido, se suman 95 casas de los judíos ${ }^{46}$. De ello se deduce que el barrio de Tarragona se encontraba entre la muralla y la judería. Este importante dato confirma que la judería no llegaba hasta la muralla de la ciudad como sucede en muchas juderías donde se aprovecha parte del muro de cierre de la ciudad como cerca del barrio judío.

\footnotetext{
${ }^{42}$ «Damus universitati de Barchinona qui voluerint populare et habitare in Valencia quintam partem domorum civitatis infra muros a porta, scilicet, de Exerea usque ad portam de Boatela [...]» (5 de junio de 1238), Llibre del Repartiment, reg. I, fol. 21.

${ }^{43}$ «[...] Omnes illas domos que sunt in çuchac Alfaz, sicut afrontat ex una parte in çuchac Çayt, quod habent homines Barchinone, de alia in çuchac Annaxar, quod habent homines Tarrachone, et de alia parte in muro civitatis et de alia parte in domibus de Azmeti, ubi afrontat çucac Axigara. II idus novembris», Llibre del Repartiment, reg. I, fol $84 \mathrm{v}$.

${ }^{44}$ «|Bartolomeo Dezpont/ [domos] Mahomat Arramat satis bonas. Mezquita de Andreu de Odena», Llibre del Repartiment, reg. III, fol. 10v.

${ }^{45}$ «In partita [h]ominum Tarachone, incipimus in mezquita de Rabat Anaxar», Llibre del Repartiment, reg. III, fol. 10v.

${ }^{46}$ «Suma hominum de Tarachone: (X)LXXXXI domus. Suma de cartis et superfluis: XXXIIII. Suma de Eclesia: II. Suma judeorum LXXXXV», Llibre del Repartiment, reg. III, fol. 12.
} 
Así pues, los atzucacs cuyas viviendas lindaban con la muralla de sur a norte eran: Çayt (habitado por hombres de Barcelona), a continuación Alzaf (habitado por 21 hombres de Lérida) y a continuación Annaxar y la partida de Tarragona. Lindando con estos por el oeste se encontraban: Con Çayt lindaba Matalhuneyax (habitado por los hombres del Puig); con Alzaf lindaba Alchesti (Ferriz Pitarch) y con Annaxar y Tarragona lindaba la judería.

Con estos datos se han localizado los diferentes atzucacs que se encontraban al Sur de la judería en el plano anteriormente elaborado. Sobre el mismo es fácil situar los baños de Amnalmelig, al lado del atzucac Alchesti en la manzana que actualmente ocupa el Real Colegio del Corpus Christi, también conocido como el Patriarca (véase fig. 1). En el mismo se sitúan los atzucacs anteriormente mencionados y la ubicación de los baños de Amnalmelig.

Para la localización del horno de Abinulliz se han analizado todas las donaciones de hornos que están reflejadas en el registro II comprobando que sólo existían tres hornos situados en los barrios limítrofes con la judería: Un horno en la plaza de la Figuera, próximo a la puerta de la judería, que en 1240 fue donado a Pedro Jordá ${ }^{47}$; un horno donado a Ramón Mirambell en 1239 ante unas casas suyas en el barrio de los hombres de la Rápita ${ }^{48}$ que, al igual que el de Tarragona, limitaba con la judería como demuestra el listado del registro III donde se señala que allí comienzan las casas de los judíos ${ }^{49}$. Asimismo, en el recuento de casas sobrantes al final del registro III se especifican conjuntamente las «del distrito de Tarragona, de los judíos y de la Rápita ${ }^{50}$; y un horno alquilado en 1239 a Jofré de Loaysa cercano a la iglesia de Santo Tomás ${ }^{51}$. Por su situación en una esquina del cuadrilátero que conforma el recinto judaico, se puede pensar que el horno de Abinulliz sea el que explotaba Jofré de Loaysa. Estaría

\footnotetext{
${ }^{47}$ Llibre del Repartiment, reg. II, fol. 36v.

${ }^{48}$ Llibre del Repartiment, reg. II, fol. 36v.

${ }^{49}$ «[...] Et remane tibi domus judeorum», Llibre del Repartiment, reg. III, fol. $12 \mathrm{v}$.

${ }^{50}$ Llibre del Repartiment, reg. III, fol. 58v.

${ }^{51}$ «Joffre de Loaysa, furnum iuxta ecclesiam Sancti Thome pro VII morabatinis alfonsinis ad Nadal» (17 de diciembre de 1239), Llibre del Repartiment, reg. II, fol. 36.
} 
situado frente a la esquina de la desaparecida calle de la Çabateria con la calle de las Avellanas. No existe documento ni donación que confirme esta hipótesis, pero sí se puede asegurar que el horno estaba situado al norte de la judería ya que el resto de hornos contabilizados en el Llibre del Repartiment están situados en otros barrios más lejanos.

Los dos últimos hitos a que hace referencia el mandato real son dos adarves que coinciden con el comienzo y el final de la delimitación del barrio judío: el adarve Abingeme y el adarve Abrahim Alvalençí.

Algunos autores hacen coincidir los dos adarves mencionados con los muros de la antigua muralla romana. El circuito del recinto romano pasaba por la calle Cabillers y giraba por Avellanas ${ }^{52}$ hacia el Norte, es decir, no llegaba hasta el barrio de la judería. Sin embargo, según los nuevos datos aportados sobre la ubicación de los baños de Amnalmelig, me inclino a pensar que estos adarves coinciden con el muro de cierre de la ciudad visigoda que por el lado Sur (adarve Abingeme) pasaba cercano a la actual calle de la Paz y el lienzo Este (adarve Abrahim Alvalençí) corría próximo a la plaza de la Figuera según el plano de Valencia visigoda de Albert Ribera y Miquel Rosellós3. Ratifica esta hipótesis el hecho de que las casas de Abrahim Alvalençí se encontraban en el barrio de Montpellier $^{54}$, colindante en este lugar a la judería ya que en este barrio se encontraba la iglesia de Santa Tecla ${ }^{55}$ situada en la plaza de la Figuera. El adarve Abingeme es la misma calle que posteriormente tomó el nombre de Call, es decir 'judería', «que todos hemos conocido con el nombre del Gallo o Gall» por corrupción de la palabra Call ${ }^{56}$.

\footnotetext{
${ }^{52}$ Carmen Aranegui Gascó, La ciudad de Valencia. Historia: la primera imagen urbana (Valencia, 2009), 67: «[...] la salida meridional a mitad de la calle Avellanas, por delante de la calle Cabillers».

${ }^{53}$ Albert Ribera i Lacomba, Miquel Roselló Mesquida, La ciudad de Valencia en época visigoda. Los orígenes del cristianismo en Valencia y su entorno (Valencia, 2000), 152.

${ }^{54}$ En el recuento de casas del barrio de los hombres de Montpellier aparece: «[...] R. Corella, domos mediocres Ebray Albalençi», Llibre del Repartiment, reg. III, fol. 13v.

${ }^{55}$ «IEcclesie/ Sancte Tecle, domos Abozehir», Llibre del Repartiment, reg. III, fol. 15v.

${ }^{56}$ «[...] últimamente se llamó del Gallo, y antes del Gall, indudablemente por corrupción de la palabra 'Call', con que es sabido se designaba el barrio que estoy deslindando», Rodrigo Pertegás, La judería de Valencia, 8 y 25.
} 
Tras la investigación llevada a cabo a la luz del Llibre del Repartiment se puede concluir que la delimitación del barrio judío llevada a cabo en 1244 era la siguiente: Partiendo del adarve Abingeme (lienzo Sur de la muralla visigoda) cercano a la actual calle de la Paz, atravesaba la actual calle de la Cruz Nueva y siguiendo por la calle Cardenal Payá llegaba a los baños de Amnalmelig junto a la Partida de Ferriç Pitarch o atzucac Achesti (actual Colegio del Corpus Christi) donde torcía hasta la puerta de la Xerea por la plaza dels Cabrerots (actual calle San Juan de Ribera) continuando por la calle de Les Medines que aún se conserva, hasta la puerta de la Xerea (actual plaza de San Vicente Ferrer) donde torcía por la calle de Christòfol Soler continuando por la desaparecida calle Çabateria hasta el horno de Abinulliz donde giraba por la calle de las Avellanas hasta el adarve de Abrahim Alvalencí (actual calle Castellvins). En este tramo se encontraba la puerta de la Figuera denominada así por la cercanía a dicha plaza ${ }^{57}$. Esta plaza estaba pavimentada con losas ${ }^{58}$ y en ella se encontraba la puerta principal de acceso al primer recinto ${ }^{59}$.

El muro dejaba fuera del barrio la calle de Christòfol Soler (medianera con el cementerio de San Juan del Hospital) y la de la Çabateria, continuación de la anterior y desaparecida en la actualidad, pero de la que pueden apreciarse vestigios en la planimetría más antigua de la ciudad (plano de Mancelli de 1608). Tras el reciente hallazgo en el trasdós de los arcosolios del cementerio medieval de San Juan del Hospital de parte de esta calle integrada en el palacio de Valeriola ${ }^{60}$,

\footnotetext{
${ }^{57}$ La puerta de la Figuera no se encontraba en la plaza del mismo nombre sino en un lugar cercano a la misma como demuestra el escrito del Consell tras el asalto a la judería de 1391: «[...] vengueren a un dels portals de la Juhería qui es prop a la plaça de la Figuera», CARBoneres, Nomenclátor de las puertas, calles y plazas de Valencia, 21.

${ }^{58}$ «[...] para que no fueran holladas las calles, habiendo merecido el contacto de este Santo martyr» (San Vicente Mártir) y en 1571 fueron usadas para pavimentar el Almudín, Orellana, Valencia antigua y moderna, tomo I, 54.

${ }^{59}$ Así se la denomina en 1239: «Jacobus Egidii, domos in açuchaco iuxta plateam de la Figera [...]», Llibre del Repartiment, reg. I, fol. 69. Siguió denominándose así «en el bando que para la primera procesión del Corpus se publicó en 1355», Orellana, Valencia antigua y moderna, tomo II, 20.

${ }^{60}$ Esta calle fue descubierta en 2005 con motivo de la elaboración del estudio previo y proyecto de puesta en valor del cementerio medieval de San Juan del Hospital llevado a cabo por los arquitectos Jorge García Valldecabres y Concepción López González.
} 
y del muro de cierre de la judería, unido a los hechos que se desencadenaron tras la ampliación del recinto se ha podido comprobar que esta calle no pertenecía al primer cierre y que fue incluida en el barrio judío tras la ampliación de 1390. Lo mismo sucede con la calle Maçana, de libre acceso, que discurría entre la puerta de la Xerea y la plaza de Cabrerots. Ambas vías eran los ejes de comunicación entre el centro de la ciudad y la salida hacia el puerto marítimo a través de la puerta de la Xerea.

Según documento firmado el 19 de septiembre de 1273, Jaime I se vio obligado a ampliar el recinto judaico hasta las casas de Ramón Castellá ${ }^{61}$ debido al gran incremento de pobladores judíos que hubo tras la conquista ${ }^{62}$. La búsqueda de este nombre en el registro I del Llibre del Repartiment confirma que sus casas lindaban con las de Guillem Soguer ${ }^{63}$. Ambos propietarios figuran en los listados de la partida de los hombres de Tarragona del registro III $^{64}$. Las casas de Ramón Castellá figuran al final del listado, muy cercanas al barrio de Xepolella situado inmediato a la puerta de la Xerea, es decir, que se encontraban al comienzo de la calle Maçana. Estos datos corroboran que la judería aumentó hacia el Este (barrio de Tarragona), concretamente hasta la calle Maçana que continuó siendo de libre acceso para la población cristiana como demuestran las protestas de los habitantes de la Xerea cuando se propone su cierre en $1390^{65}$.

\footnotetext{
${ }^{61}$ Hinojosa Montalvo, La judería de Valencia en la Edad Media, 422.

${ }^{62}$ Este incremento fue debido a las buenas condiciones ofertadas por el monarca para aquellos judíos que viniesen a repoblar la ciudad.

${ }^{63}$ «R. Castellanus, domos pro stabulo contiguas aliis domibus tuis, et fuerunt G. Soger [...]», Llibre del Repartiment, reg. I, fol. 72.

${ }^{64}$ «Guillem Soguer de Rocafera, II bonas domos Ali Adeni, Juçef Anfetech II/ Michel Homodei, domos Juçef Gober Abenfateri/ Rodericus Castellan, domos Amet Almançafi», Llibre del Repartiment, reg. III, fol. 12.

${ }^{65}$ Denominada en la Proposición hecha al Consejo General de la ciudad el 4 de junio de 1390 en la que se exponen los problemas que creará el cierre de la judería: «Dun cap de carrer prop, e dins del Portal de la Xerea, per lo cual carrer solía hom anar á la Plaça apelladada del Cabrerots e al carrer de En-Maçana», Orellana, Valencia antigua y moderna, tomo I, 609.
} 
Es muy probable que en esta ampliación de 1273 se construyera la puerta denominada «dels Cabrerots» ${ }^{66}$ en la plaza del mismo nombre ${ }^{67}$ al ser incluida la calle de les Medines en el nuevo recinto. Tosca, en su plano de 1704 sitúa la plaza de Cabrerots entre la calle de les Medines y la calle Carn y Cols, perdurando hasta finales del siglo XIX con la apertura de la calle de la Paz. Algunos autores, como el propio Rodrigo Pertegás ${ }^{68}$, hacen coincidir esta plaza con la denominada Cruz Nueva en la actualidad. Parece más plausible la propuesta de Tosca ya que la puerta de la Xerea y la de Cabrerots se encontraban unidas por la desaparecida calle de En Maçana que tenía su inicio en la actual plaza de San Vicente Ferrer, donde estaba la puerta de la Xerea, y finalizaba precisamente donde Tosca establece la plaza dels Cabrerots. Ratifica esta localización de la plaza dels Cabrerots la descripción que el Patriarca San Juan de Ribera hace de los límites del Colegio del Corpus Christi en la escritura de fundación:

[...] en la parroquia del glorioso apóstol San Andrés en la calle vulgarmente dicha la Calle de la Nao ante la plaza de las Esquelas y estudio general de la dicha ciudad que terna y ha de tener de ámbito de una parte dentro del cabo de la calle que baxa de la dicha plaça de las Esquelas hasta la plaza de la Cruz Nueva prosigue hasta otra calle que de las espadas de la dicha Cruz Nueva sube a la plaza de las dichas Esquelas, la plaza dicha de los Cabrerots y de dicha plaça a la calle de la Nao delante del horno vulgarmente dicho de Na Plana o del estudio general $[\ldots]$.

Es decir, en esta descripción la plaza dels Cabrerots no coincide con la de la Cruz Nueva sino con la misma que grafía el padre Tosca.

A partir de estos datos es posible reconstruir el límite de esta ampliación de la judería hasta llegar a la calle Maçana.

\footnotetext{
${ }^{66}$ Rodrigo Pertegás confirma la existencia de este portal por el acta de una visita pastoral girada en 1388 a la parroquia de San Andrés en la que se lee que un hombre vive cerca del portal con esposa y concubina: «Item en moya lo traginer qui miratur prope portale juderie habet uxor et concubina» (Rodrigo Pertegás, La judería de Valencia, 7).

${ }^{67}$ Según consta en un listado de avecindamiento de 1389.

${ }^{68}$ Rodrigo Pertegás, La judería de Valencia, 24-25.
} 


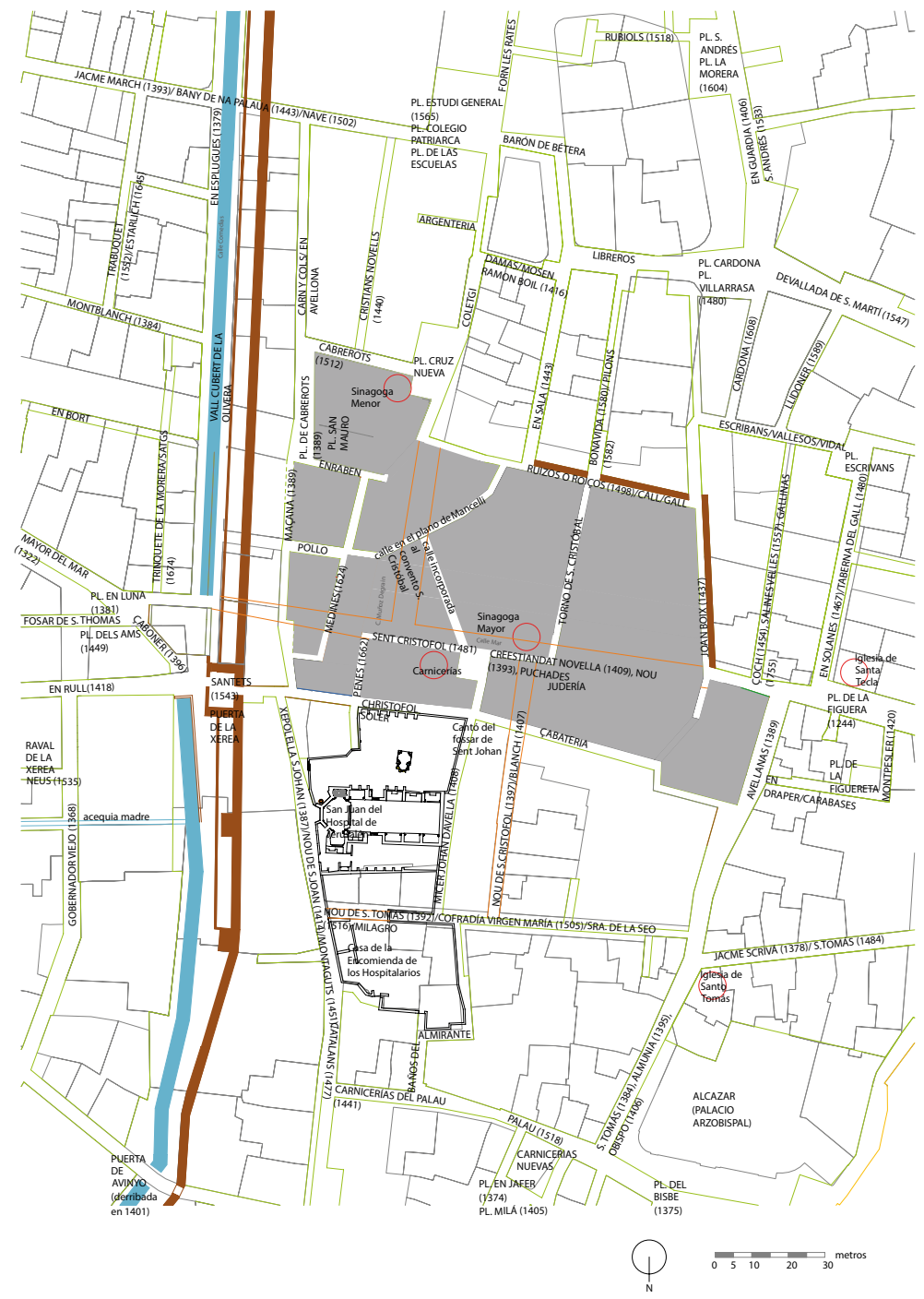

Fig. 2. Plano de la Juheria Vella y su entorno inmediato donde se ha rotulado el nombre de las calles y el año en que han recibido dicha denominación. Sobre el parcelario actual se dibujan las manzanas edificatorias según los planos de Mancelli (1608) y Tosca (1704). Se han grafiado las calles que fueron abiertas tras el asalto de 1391. Reproducción en color del plano en la versión electrónica del artículo (http://sefarad.revistas.csic.es). 


\section{2.- PRINCIPAles CAlles DEL PRIMER RECINTO DE LA JUDERÍA Y SU EN- TORNO INMEDIATO}

De este recinto o Juheria Vella la única calle que conserva parte de su trazado así como su denominación es la calle de les Medines ${ }^{69}$.

Existe otra calle, cerrada en la actualidad y convertida en patio interior de un edificio de viviendas denominada del Cristo de las Penas o «carrer de les Penes» ${ }^{70}$ tal como lo rotula Tosca en su plano de 1704. Esta calle unía el cementerio de San Juan del Hospital con la calle de la Carnicería del barrio judío (absorbida por la actual calle del Mar). Durante el primer periodo de la judería debió estar cerrada en su unión con el conjunto hospitalario y probablemente a través de ella se realizaba el acceso lateral o secundario a las carnicerías judías.

La calle que transcurría entre la parcela de los sanjuanistas y la judería se denominaba Cristòfol Soler. Fue cerrada y anexionada a la parcela del palacio de Valeriola y aún se conserva en el interior del palacio. Tiene una anchura de 10 palmos valencianos (2’30 m) y en ella todavía subsiste el muro trasero de los arcosolios del cementerio medieval de San Juan del Hospital actuando como muro de cierre de la judería. Está construido con la técnica del tapial, con una altura de 22 palmos $(5 \mathrm{~m})$ y un grosor de 2 palmos $(46 \mathrm{~cm})$. El muro fue restaurado y reforzado al incorporarse como parte del palacio de Valeriola mediante un recubrimiento de fábrica de ladrillo macizo.

Esta calle finalizaba en el «cantó del fossar de Sent Joan» (esquina con la calle de Micer Johan Davella) donde pasaba a denominarse calle de la Çabateria $^{71}$ hasta llegar a la calle de las Avellanas. La calle que en el año

\footnotetext{
${ }^{69}$ Definida en «Deliveración de la Ciudad» el 17 de Mayo de 1659 con motivo de la venta de las Carnicerías: «el pati que solía ser casa baixa y escaleta situada en la present ciutat, Parroquia de Sanct Thomas en lo carrer dit de les Medines, que es la travesa per hon se entra per les cuatre cantonades del carrer de la Mar, a hon está el forn dit de Sanct Christòfol, pero lo cual se va á la Creu Nova, y a ma Esquerra a la Plaça de la Olivera», cf. Orellana, Valencia antigua y moderna, tomo II, 262.

${ }^{70}$ Esta denominación tiene su origen en la procesión que por ella pasaba el Jueves Santo transportando la imagen del Cristo de las Penas venerado en una de las capillitas del tránsito de acceso a la iglesia sanjuanista.

${ }^{71}$ Existe un escrito de 26 de noviembre de 1377a raíz de una querella donde aparece esta calle (Archivo del Reino de Valencia [= ARV], Bailía, ap. 127, fol. 156r). Asimismo aparece en una lista de avecindamiento de 3 de noviembre de 1391 (AMV, Llibres de
} 


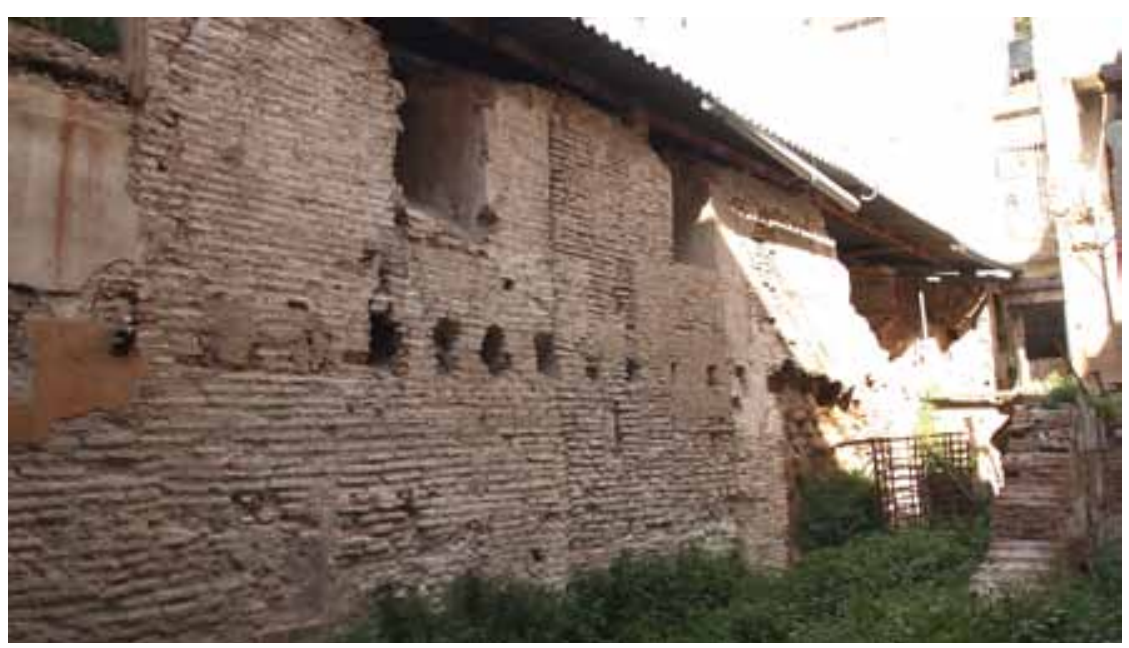

Fig. 3. Muro trasero de los arcosolios del cementerio medieval de San Juan del Hospital que conformaba la calle de Cristòfol Soler, actualmente incorporada a la parcela del palacio de Valeriola.

1408 es denominada de Micer Johan Davella en el Llibre de Sotsobreria de Murs y Valls $^{72}$ es probablemente la misma que Luis Gascó Pascual ${ }^{73}$ denomina calle del Atrio. Se iniciaba en el callejón sin salida que en 1393 fue convertido en la actual calle del Milagro y salía a la plaza de la carnicería de los judíos.

Esta calle fue cerrada y anexionada a la parcela de San Juan del Hospital en 1428. El recorrido de esta calle aún es reconocible sobre el parcelario actual; en el plano de Mancelli figura como un callejón sin salida y en el grabado de José Fortea de 1738 del original de Tosca aparece como patio interior.

Paralela a esta existía una calle sin salida que en 1397, tras la conversión de la sinagoga Mayor en convento de San Cristóbal, se denominó de

Aveinaments, b3-11, fol. 190v), cit. Hinojosa Montalvo, La judería de Valencia en la Edad Media, 437, nota 850.

${ }^{72}$ CARBoneres, Nomenclátor de las puertas, calles y plazas de Valencia, 72.

${ }^{73}$ Luis Gascó Pascual, La iglesia de San Juan del Hospital de Valencia (Valencia 1969; reimpr. Valencia 1998), 41. 


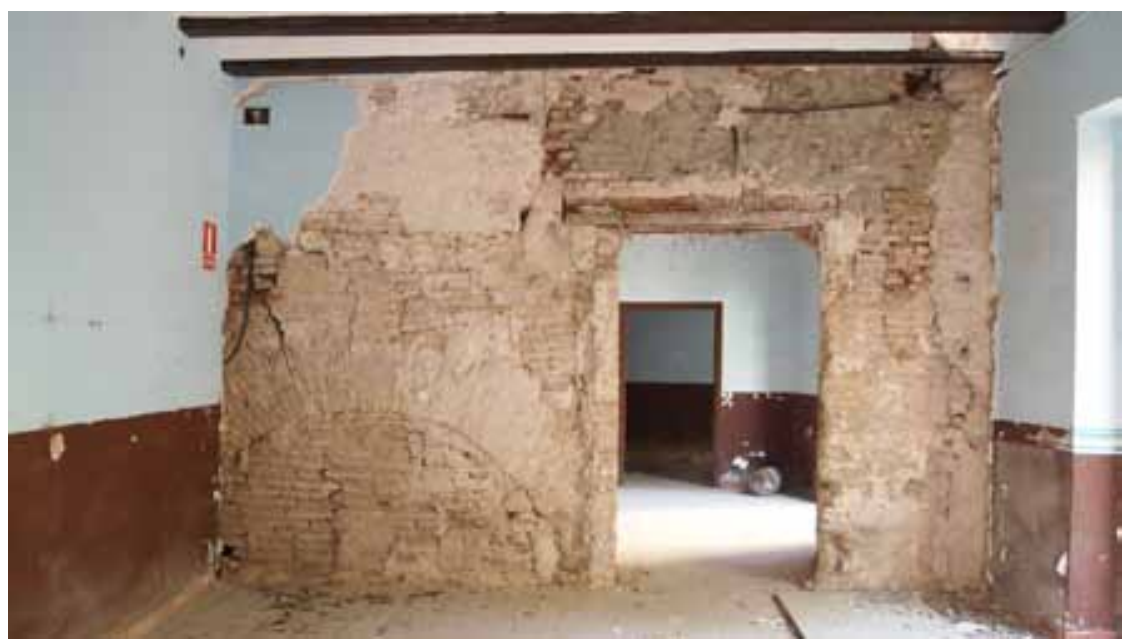

Fig. 4. Arco de ladrillo macizo perteneciente a la fachada de un edificio de la antigua calle de Micer Johan Davella, actualmente incorporada en casi todo su recorrido a la parcela de San Juan del Hospital. Restos hallados en las casas colindantes al palacio de Valeriola. En la confluencia de esta calle con la calle de Cristòfol Soler, en el lugar que se denominaba «fossar de Sent Joan, se encontraba el portal denominado por Rodrigo Pertegás (en su Plano probable de la judería de Valencia) la puerta de la Çabateria por la que los cristianos tenían acceso directo a las carnicerías para adquirir la carne que los judíos no consumían.

San Cristóbal. Posteriormente se denominó calle de Blanch ${ }^{74}$ según una nómina de avecinamiento de 1407. Al ser anexionada la calle de Micer Johan Davella al conjunto sanjuanista este callejón sin salida se convirtió en calle (actual de San Cristóbal) que en 1419 pasa a llamarse Carrer Nou de Sent Cristòfol como la rotula Tosca en su plano de 1704. Sin embargo Orellana afirma que este Carrer Nou era el tramo de calle del Mar entre la plaza de Santa Tecla y el convento de San Cristóbal ${ }^{75}$.

La calle Xepolella ${ }^{76}$ se encontraba al inicio de la actual calle de Trinquete de Caballeros pero con diferente inclinación a la actual. La inclinación

\footnotetext{
${ }^{74}$ CARBONERES, Nomenclátor de las puertas, calles y plazas de Valencia, 37.

${ }^{75}$ Se menciona en una escritura de 1596 «y sin duda es el trecho de la calle del Mar hacia dichas monjas, por lo que se abrió dicha calle ó trecho despues de la expulsión de los Judíos», cf. ORellana, Valencia antigua y moderna, tomo II, 354.

${ }^{76}$ «[...] transformación cristiana de Subiliya, así llamada porque estaba ubicada allí la mezquita de aquel nombre, junto a la puerta de la Exarea», cf. Vicente Coscollá SANZ, La Valencia musulmana (Valencia, 2003), 68.
} 

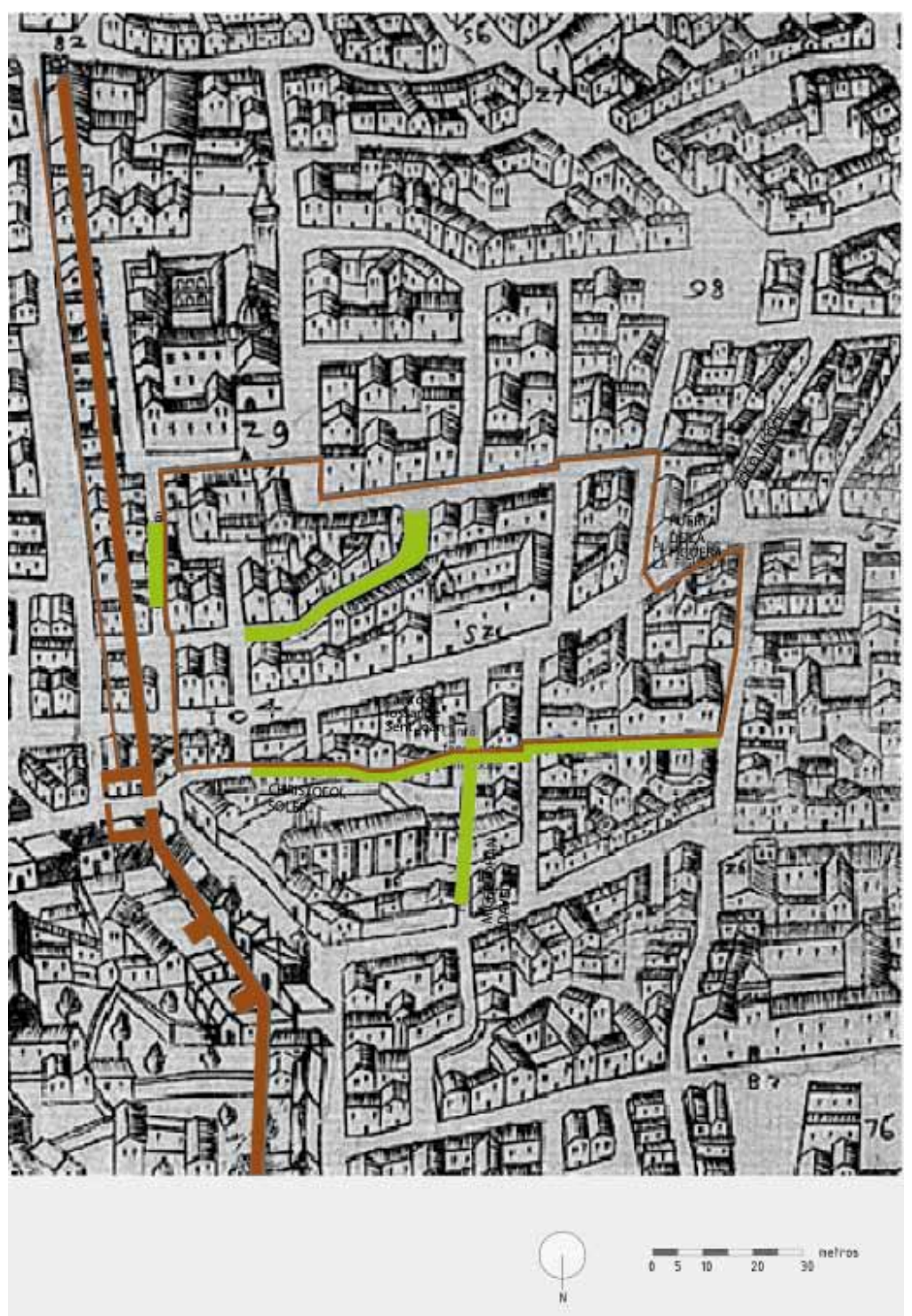

Fig. 5. La Juheria Vella sobre el plano de Mancelli (1608). Se señalan las calles que han desaparecido, de las que aún quedan vestigios en este plano. Reproducción en color del plano en la versión electrónica del artículo (http://sefarad.revistas.csic.es). 
todavía se adivina en los restos del muro que cerraba la parcela sanjuanista en la actual calle de Trinquete de Caballeros. También se la denominó posteriormente calle de Catalans según consta en documentos de $1377^{77} \mathrm{y}$ la nómina de avecinamiento de $1477^{78}$. Otra calle que lindaba con el primer recinto es la calle de las Avellanas ${ }^{79}$ en el tramo comprendido entre la desaparecida calle de la Çabateria y la actual calle del Mar (el otro trecho se denominaba calle de Santo Thomas). En la intersección de la calle de la Çabateria y la calle de las Avellanas, donde se encontraba el portal de la Çabateria, se producía un ensanchamiento de esta última que aún persiste, denominado plaza de las Avellanas. También se la ha denominado Plaça de Marrades por estar allí la casa de los Masas de Lisana del linaje de Marradas y anteriormente fue de los Duques de Mandas por lo que la plaza también recibió este nombre. Desde 1401 y posteriormente en los años 1407 y 1434 está documentada la calle denominada «de la Juheria» situándola frente a la iglesia de Santo Tomás ${ }^{80}$. Podría tratarse de la calle «de la Çabateria», que a raíz de asalto sufrido en 1391 cambió su nombre por este otro recordando el lugar en el que anteriormente estuvo el barrio judío.

Una escritura de 18 de Enero de 1599 describe la calle de Carnicols como perteneciente a la judería ${ }^{81}$. Según Orellana, la calle de Carn y Cols ${ }^{82}$, también denominada «Na Abellona» según escritura de 1467, comenzaba «en la Plazuela de las Carnicerías de San Christoval y torciendo a cabo, frontis, baja á una Plaza llamada ahora de 'San Mauro' (que está a espaldas del Colegio del Patriarca, y á espaldas también de la 'Cruz Nueva') y cuya calle de Carnicol sigue y continua con el mismo nombre hasta la calle de la Nave» junto al baño de Na Palaua. Tosca también la denomina de «Carn

\footnotetext{
${ }^{77}$ ARV, Bailía, apéndice 127, fol. 174v, cit. Hinojosa Montalvo, La judería de Valencia en la Edad Media, 437.

${ }^{78}$ CARBoneres, Nomenclátor de las puertas, calles y plazas de Valencia, 42.

${ }^{79}$ Aparece con este nombre en un bando publicado en 24 de noviembre de 1423 y en un documento de 1392, cf. Orellana, Valencia antigua y moderna, tomo I, 126.

${ }^{80}$ Hinojosa Montalvo, La judería de Valencia en la Edad Media, 438.

${ }^{81}$ «Plaza de Cornicols, olim la Juheria, en la parroquia de San Andrés», cf. ORELLANA, Valencia antigua y moderna, tomo I, 357.

${ }^{82}$ Según escritura de 8 de Abril de 1467 se la denomina calle de «Na Abellona», cf. Orellana, Valencia antigua y moderna, tomo I, 7. Según otra escritura ante Alonso Ayerbe de 10 de junio de 1497 se la denomina también como calle de «En Avellona, qua tenditur del forn del Argentera la Juheria», cf. Orellana, Valencia antigua y moderna, tomo I, 564.
} 
y Cols» en su plano de 1704. La plaza de las Carnicerías de San Cristóbal a la que hace referencia Orellana es la misma que también se denominó de «Carn y Col» $\mathrm{y}$ «dels Cabrerots». La denominación «Carn y Col» coincide con el sobrenombre que recibe un judío, Carnicols, en el siglo XIV ${ }^{83}$. La calle que actualmente se denomina de la Cruz Nueva se denominaba en el momento de la conquista calle Algalga por los datos extraídos del Llibre del Repartiment expuestos en el apartado anterior. En el lugar que ahora ocupa el Colegio del Corpus Christi existía un callejón sin salida denominado Argentería 84. La calle que unía la plaza dels Cabrerots con la puerta de la Xerea era la calle Maçana o En Maçana.

Existía otra calle, denominada del Call, en el interior de este primer recinto que Rodrigo Pertegás menciona en su opúsculo aunque Carboneres no recoge este nombre sino el de Roiços según una nómina de avecindamiento de 1498 y Tosca la rotula «dels Ruizos».

La calle En Sala recibe este nombre en la lista de avecindamiento de 1443 y Tosca la rotula así en su plano y ha mantenido dicha denominación hasta nuestros días.

La calle de En Joan Boix (mencionada en la lista de avecindamiento de 1437) es la que en 1524 se denomina «de Castellvins» ${ }^{85}$. Comenzaba en la actual calle Libreros y desembocaba en la actual calle del Mar. En la lista de avecindamiento de 1574 es denominada como calle de Benavi$\operatorname{des}^{86}$. Actualmente se denomina calle de Ruiz de Lihory. En esta calle se instaló un judío aragonés, Azarías, abuelo de Luis de Santángel.

En el interior del recinto judaico existía una calle de trazado bastante tortuoso según se aprecia en el plano de Mancelli, que partiendo de la calle de les Medines transcurría por detrás del convento de San Cristóbal para desembocar en la calle del Call o del Gall como se la llamó posteriormente. Parte de esta calle y otra que partía de enfrente de las carnicerías son incorporadas en 1692 al convento de San Cristóbal, razón por la

\footnotetext{
${ }^{83}$ Un «fill de Carnicols» figura en la nómina de multas impuestas en 1383 por el baile general a diversos judíos, cf. José Ramón Magdalena Nom de Déu, «Delitos y 'calònies' de los judíos valencianos en la segunda mitad del siglo XIV (1351-1384)», Anuario de Filología 2 (1976), 181-225: 206.

${ }^{84}$ Rodrigo Pertegás, La judería de Valencia, 25.

${ }^{85}$ CARBoneres, Nomenclátor de las puertas, calles y plazas de Valencia, 42.

${ }^{86}$ CARBoneres, Nomenclátor de las puertas, calles y plazas de Valencia, 38.
} 
cual los jurados de la ciudad obligan a las monjas canongesas a abrir una calle que conecte la del Mar con la plaza de la Cruz Nueva ${ }^{87}$. La denominación de esta vía desaparecida se desconoce pero la nueva calle recibe el nombre de San Carlos según consta en el plano de Tosca. Actualmente es la calle de Muñoz Degrain.

La plaza de les Abaccots figura en un documento de 1377 especificando que un cristiano vive en este lugar de la judería valenciana ${ }^{88}$ pero se desconoce su ubicación.

En el norte de la judería, cerca del muro de cierre, se encontraba la plaza que Rodrigo Pertegás denomina de la Carnicería ${ }^{89}$ por hallarse en ella las carnicerías judías (actual palacio de Valeriola medianero con la antigua cerca del barrio judío). Desde esta misma plaza se accedía a la sinagoga Mayor, convertida en 1406 en convento de San Cristóbal.

La calle del Çoch aparece rotulada en el plano de Tosca coincidiendo con la que actualmente es un callejón sin salida con entrada desde la calle del Mar denominado de las Gallinas. Según Carboneres ${ }^{90}$ se tiene noticia de ella por la lista de avecindamiento del año 1454. El Açòch o 'zoco' era una calle comercial que, según José Ramón Magdalena Nom de Déu tenía una doble función «artesanal y comercial, se vendía y fabricaba en los obradores, ocupados por peleteros, sederos, tintoreros, plateros, e incluso un herrero» ${ }^{91}$. Los obradores eran alquilados tanto por cristianos como por judíos. Era propiedad de la Corona y cada año salían a subasta los puestos. Entre 1357 y 1365 se observa un declive quedándose algunos obradores sin adjudicar. También tuvo el nombre de «En Coch» según la lista de avecindamiento de $1473^{92}$. El Padre Tosca, en su plano de 1704 le da a esta calle el nombre de «calle de les Salines Velles olim Çòch». Este nombre rememora la purificación y venta de sal que se realizaba en uno de los locales del lado izquierdo. Se trataba de un pasaje cerrado al que se accedía desde las inmediaciones de la puerta de la Figuera. Según

\footnotetext{
${ }^{87}$ Rodrigo Pertegás, La judería de Valencia, 21.

${ }^{88}$ Hinojosa Montalvo, La judería de Valencia en la Edad Media, 438.

${ }^{89}$ Rodrigo Pertegás, La judería de Valencia, 24.

${ }^{90}$ CARBoneres, Nomenclátor de las puertas, calles y plazas de Valencia, 44.

${ }^{91}$ Magdalena Nom de Déu, «Un zoco judaico en la Valencia medieval», 318.

${ }^{92}$ CARboneres, Nomenclátor de las puertas, calles y plazas de Valencia, 52.
} 
Leopoldo Piles Ros ${ }^{93}$ nunca estuvo incluido en el recinto cerrado de la judería y así se confirma en documento de 1333 cuando el rey realiza una concesión de tres obradores «in vico publico vocato del Açoch, prope judaria civitates ${ }^{94}$ y en la visita pastoral girada a Santo Tomás ${ }^{95}$ en 1354. Cuando en 1390 se amplía el perímetro del barrio judío, tampoco llega a incorporarse el Açoch al nuevo recinto ya que en el libro de cuentas de las obras realizadas no existen asientos de gastos en la puerta de la Figuera, es decir, que la puerta no se traslada hacia el oeste para incluir la entrada a la calle del Açoch en el nuevo perímetro. Ratifica esta hipótesis el hecho de que el Açoch no sufre daños ${ }^{96}$ tras el asalto de 1391 que se llevó a cabo en gran parte por la puerta de la Figuera. Sin embargo, al encontrarse su acceso junto a esta puerta ${ }^{97}$ y ser explotado en su mayoría por comerciantes y artesanos judíos, se le ha denominado en ocasiones «Açoch judío» ${ }^{98}$.

Recibido: 20/05/2013

Aceptado: 07/05/2014

${ }^{93}$ Leopoldo PILES Ros, «El Açoch de la aljama judaica de Valencia», Sefarad 45 (1985), 69-130: 75.

${ }^{94}$ Archivo de la Corona de Aragón [= ACA], Cancillería [= C], reg. 846, fol. 202r-v, cf. Piles Ros, «El Açoch de la aljama judaica de Valencia», 77.

${ }^{95}$ Preguntados si el zoco pertenecía a la judería «dixerunt quod extrea juderiam», Archivo de la Curia Eclesiástica de Valencia, Visitas, tomo II, fol. 28, cit. RodRIGo Pertegás, La judería de Valencia, 7.

${ }^{96}$ Piles Ros, «El Açoch de la aljama judaica de Valencia», 77.

${ }^{97}$ Jaime I concede un obrador «in loco vocato lasoch» situado a la entrada de la judería, cf. Magdalena Nom de Déu, «Un zoco judaico en la Valencia medieval», 309.

${ }^{98}$ En la cesión enfitéutica confirmada en 1327 por Jaime II de tres obradores a un portero real aparece el término «açocho judeorum» (ACA, C, reg. 230, fol. 77), cf. Magdalena Nom de Déu, «Un zoco judaico en la Valencia medieval», 309. 